\title{
DISEÑO DE SECCIONES SOMETIDAS A FLEXIÓN COMPUESTA, CON GRAN EXENTRICIDAD SEGÚN EL REGLAMENTO ACI 318-02
}

Fernando Perdomo*

\section{RESUMEN:}

Descripción de los cambios más significativos en el nuevo Reglamento de las Construcciones para Hormigón Estructural ACI 318-02, en lo referente al diseño de secciones de hormigón armado sometidas a flexión y flexión compuesta con gran excentricidad.

Se presenta la deducción de las fórmulas básicas para el diseño de secciones de hormigón armado usando los nuevos criterios introducidos en el Reglamento, así como las nuevas tablas de coeficientes para diseño.

\section{PALABRAS ClAVES:}

Hormigón armado, concreto reforzado, diseño estructural, Reglamento $\mathrm{ACI}$

\section{INTRODUCCIÓN}

La nueva versión 2002 del ACI 318 contiene los mayores y más fundamentales cambios hechos al Reglamento en casi 40 años. En este artículo vamos a estudiar con detalle cómo estos cambios han afectado los procedimientos de diseño de secciones rectangulares sometidas a flexión compuesta con gran excentricidad.

" Samuel S. Conde \& Asociados, Santo Domingo, República Dominicana 
Los cambios más significativos son la eliminación de los conceptos "Diseño como Viga" y "Diseño como Columna", y la eliminación del concepto de "Cuantía Máxima". En la nueva versión sólo se tienen elementos sometidos a flexión compuesta. Según el capitulo 10.3 "Principios y Requisitos Generales", cuando la excentricidad es muy grande, como en el caso de las vigas, se trata de secciones con "tracción dominante" ( $\$ 10.3 .4)$, y en el caso de excentricidades pequeñas, como el caso de las columnas, se trata de secciones con "compresión dominante" $(\S 10.3 .3)$. A diferencia de las versiones anteriores, la definición del tipo de solicitación (tracción o compresión dominante) no se hace a partir de la magnitud de la fuerza axial, sino de la deformación unitaria de la armadura traccionada.

En ambos casos, el procedimiento de diseño es el mismo: se debe demostrar que las fuerzas interiores producidas por las cargas factorizadas son menores o iguales que las fuerzas nominales de rotura de la sección multiplicadas por el factor de reducción de resistencia $\varnothing$. En general tendremos:

$$
\begin{aligned}
& \mathrm{Mu}<=\emptyset^{*} \mathrm{Mn} \\
& \mathrm{Nu}<=\emptyset^{*} \mathrm{Nn}
\end{aligned}
$$

En las versiones anteriores del reglamento, el control de deformaciones en las armaduras traccionadas se hacía a través del control de la cuantía. Al limitar la cuantía a una fracción de la cuantía de la sección balanceada (cuantía máxima), lo que se hacía era asegurar una deformación en las armaduras traccionadas mayor que la deformación de fluencia. En esta nueva versión del Reglamento no se limita la cuantía, sino que el control de las deformaciones se hace a través del factor $\emptyset$, el cual depende directamente de la deformación del acero, penalizando los casos donde las deformaciones del acero son pequeñas con valores menores de $\varnothing$. 


\section{FACTORES DE CARGA}

Para obtener las cargas factorizadas o cargas de rotura, se beben multiplicar las cargas de servicio por los factores de carga (§9.2). A continuación se presenta un resumen de las principales combinaciones:

\begin{tabular}{|l|l|}
\hline Combinación & Resistencia Requerida \\
\hline Carga muerta (D) y carga viva $(\mathrm{L}):$ & $1.4 \mathrm{D}$ \\
& $1.2 \mathrm{D}+1.6 \mathrm{~L}$ \\
\hline Carga muerta $(\mathrm{D})$, carga viva $(\mathrm{L})$ & $1.2 \mathrm{D}+1.6 \mathrm{Lr}+0.8 \mathrm{~W}$ \\
y Viento $(\mathrm{W}):$ & $1.2 \mathrm{D}+1.0 \mathrm{~L}+1.6 \mathrm{~W}$ \\
& $0.9 \mathrm{D}+1.6 \mathrm{~W}$ \\
\hline Carga muerta (D), carga viva $(\mathrm{L})$ & $1.2 \mathrm{D}+1.0 \mathrm{~L}+1.0 \mathrm{E}$ \\
y Sismo $(\mathrm{E}):$ & $0.9 \mathrm{D}+1.0 \mathrm{E}$ \\
\hline
\end{tabular}

La lista completa de combinaciones de carga es como sigue $(\S 9.2)$ :

Ecuación 9-1: $\quad U=1.4(D+F)$

Ecuación 9-2: $\quad U=1.2(D+F+T)+1.6(L+H)$

Ecuación 9-3: $\quad U=1.2 D+1.6(L r o ́ s ~ o ́ R)+(1.0 L$ o $0.8 W)$

Ecuación 9-4: $\quad U=1.2 \mathrm{D}+1.6 \mathrm{~W}+1.0 \mathrm{~L}+0.5(\mathrm{Lr}$ ó $\mathrm{S}$ ó $\mathrm{R})$

Ecuación 9-5: $\quad U=1.2 \mathrm{D}+1.0 \mathrm{E}+1.0 \mathrm{~L}+0.2 \mathrm{~S}$

Ecuación 9-6: $\quad \mathrm{U}=0.9 \mathrm{D}+1.6 \mathrm{~W}+1.6 \mathrm{H}$

Ecuación 9-7: $\quad U=0.9 D+1.0 E+1.6 \mathrm{H}$

Nomenclatura:

D: Carga muerta Lr: Carga viva de techo

L: Carga viva R: Lluvia

E: Sismo T: Temperatura, asentamientos, cambios de volumen

W: Viento

F: Presión de fluidos S: Nieve

$\mathrm{H}$ : Peso y/o empuje de tierra 
El factor de carga para L (carga viva) en las ecuaciones 9-3 a 9-5 se puede reducir a 0.5 excepto en garajes, áreas ocupadas como lugares públicos de reunión y todas las áreas donde la carga viva sea mayor o igual a $500 \mathrm{~kg} / \mathrm{m}^{2}$.

Donde las cargas sísmicas sean cargas de servicio, se debe usar 1.4E en vez de 1.0E en las ecuaciones 9-5 y 9-7.

\section{FACTOR DE REDUCCIÓN DE RESISTENCIA $\varnothing(§ 9.3 .2)$}

El valor del factor de reducción de resistencia $\varnothing$ ya no depende de la Fuerza Axial Nu, sino de la deformación del acero más alejado del borde comprimido.

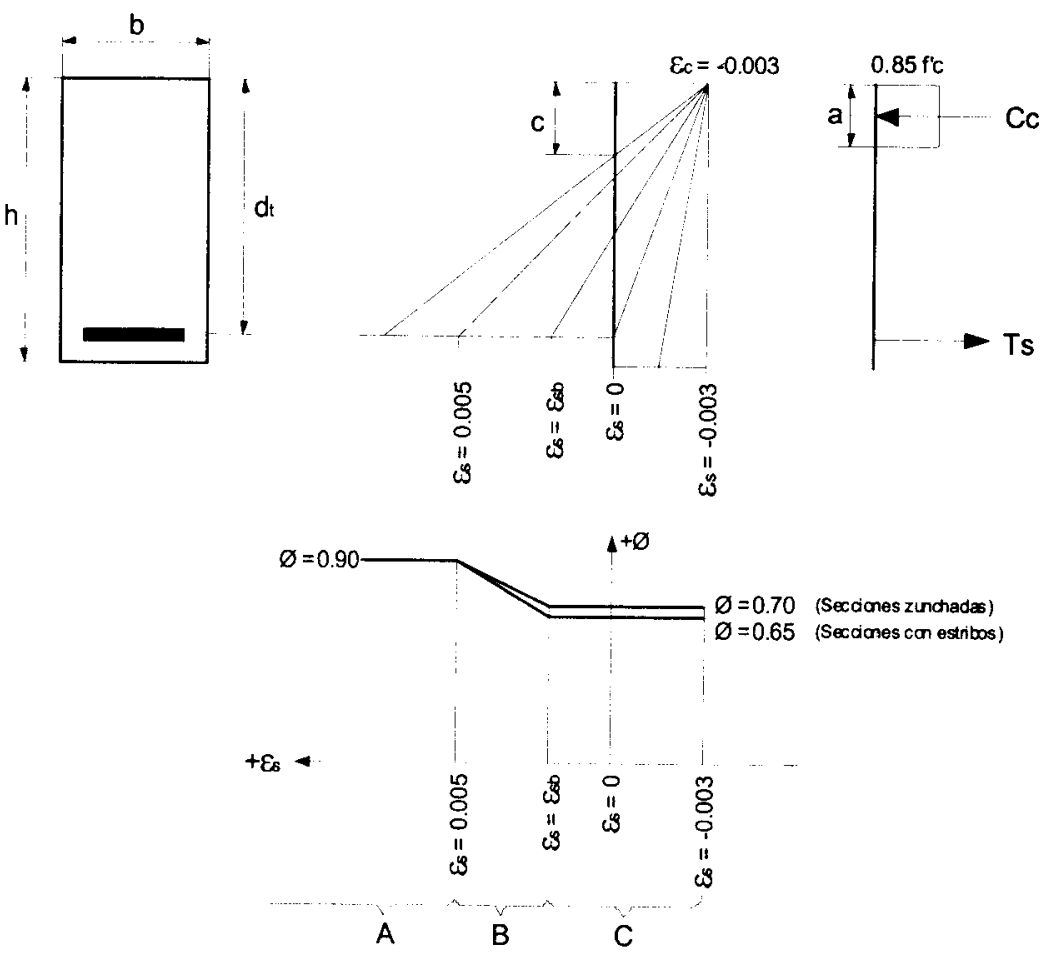


Valores de Ø:

Zona A: Tracción dominante: $\quad \varepsilon_{s} \geq 0.005 \varnothing=0.90$

Zona B: Zona de transición: $\quad 0.005 \geq \varepsilon_{s} \geq \varepsilon_{s b}$

$\varnothing=0.65+0.25^{\star}\left(\varepsilon_{\mathrm{s}}-\varepsilon_{\mathrm{sb}}\right) /\left(0.005-\varepsilon_{\mathrm{sb}}\right) \quad$ (Sección con estribos)

$\varnothing=0.70+0.20^{*}\left(\varepsilon_{\mathrm{s}}-\varepsilon_{\mathrm{sb}}\right) /\left(0.005-\varepsilon_{\mathrm{sp}}\right) \quad$ (Secciones zunchadas)

$\varepsilon_{s b}=f_{y} / E_{s}: \quad$ Deformación de fluencia del acero

( $=0.002$ para acero de pretensado)

Para $f_{\mathrm{y}}=4.2$ ton $/ \mathrm{cm}^{2}$ o para acero de pretensado $\left(\varepsilon_{\mathrm{sb}}=0.002\right)$ :

$\varnothing=0.4833+83.33^{*} \varepsilon_{\mathrm{s}} \quad$ (Secciones con estribos)

$\varnothing=0.5667+66.67^{\star} \varepsilon_{\mathrm{s}} \quad$ (Secciones zunchadas)

Zona C: Compresión dominante: $\varepsilon_{\mathrm{s}} \leq \varepsilon_{\mathrm{sb}}$

$\varnothing=0.65$

(Secciones con estribos)

$\varnothing=0.70$

(Secciones zunchadas)

4. PLANTEAMIENTO GENERAL PARA ZONAS A Y B:
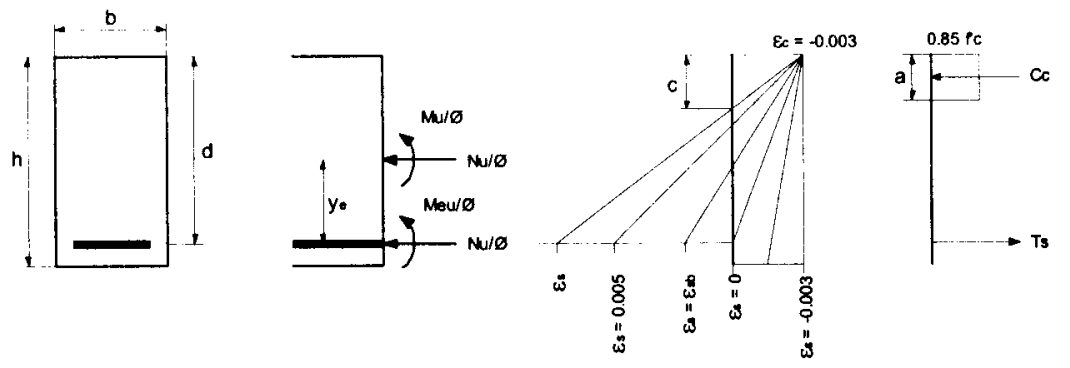

$$
\begin{array}{ll}
M_{e u}=M_{u}+N_{u}{ }^{*} y_{e} & y_{e}=d-h / 2 \quad+N_{u}=\text { compresión } \\
k_{c}=c / d & k_{a}=a / d
\end{array}
$$


Posición del eje neutro: (deformaciones con su signo: $\varepsilon_{c}=-0.003$ ) $\left(\varepsilon_{\mathrm{s}}-\varepsilon_{\mathrm{c}}\right) / \mathrm{d}=-\varepsilon_{\mathrm{c}} / \mathrm{c}$

de donde $\quad \mathrm{c} / \mathrm{d}=\mathrm{k}_{\mathrm{c}}=-\varepsilon_{\mathrm{c}} /\left(\varepsilon_{\mathrm{s}}-\varepsilon_{\mathrm{c}}\right) \quad$ (Ecuación 4.1)

o sea:

$$
c=k_{c}^{*} d
$$

Altura del bloque de compresión:

$$
\begin{aligned}
& a=b_{1}{ }^{*} \mathrm{c}=\beta_{1}{ }^{*} k_{c}{ }^{*} d \quad k a=\beta_{1}{ }^{*} k_{c} \\
& a=k_{a}{ }^{*} d \\
& \mathrm{f}^{\prime} \mathrm{c}>280 \mathrm{~kg} / \mathrm{cm}^{2}: \beta_{1}=0.85-\left(\mathrm{f}^{\prime} \mathrm{c}-280\right) / 1400 \geq 0.65
\end{aligned}
$$

Condiciones de equilibrio:

$$
\begin{aligned}
& C_{c}=0.85^{\star} f^{\prime}{ }_{c}^{\star} a^{\star} b \\
& \text { Ts }=f_{s}^{*} A_{s}
\end{aligned}
$$

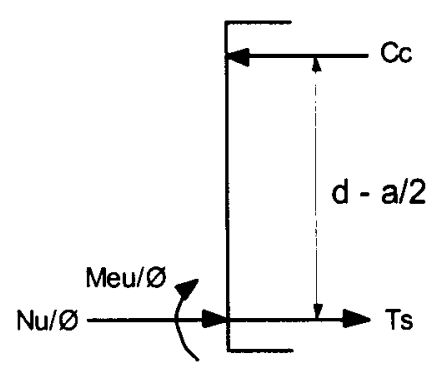

a) $\Sigma N=N_{u} / \varnothing+T_{s}-C_{c}=0$

b) $\Sigma M_{\mathrm{T}}=\mathrm{M}_{\mathrm{eu}} / \varnothing-\mathrm{C}_{\mathrm{c}}{ }^{*}(d-\mathrm{a} / 2)=0$

c) $\Sigma M_{\mathrm{c}}=\mathrm{M}_{\mathrm{eu}} / \varnothing-\left(\mathrm{N}_{\mathrm{u}} / \varnothing+\mathrm{T}_{\mathrm{s}}\right)^{*}(\mathrm{~d}-\mathrm{a} / 2)=0$

de la ecuación (b):

$$
\begin{aligned}
& M_{e u}=\varnothing^{*} C_{c}^{*}(d-a / 2)
\end{aligned}
$$

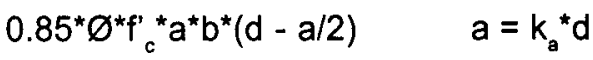

$$
\begin{aligned}
& 0.85^{*} \varnothing^{*} f_{c}{ }_{c}^{*} b^{*} d^{2 *} k_{a}{ }^{*}\left(1-k_{a} / 2\right) \\
& M_{e u} /\left(f_{c}{ }_{c}^{*} b^{*} d^{2}\right)=0.85^{\star} \emptyset^{*} k_{a}{ }^{*}\left(1-k_{a} / 2\right)
\end{aligned}
$$


haciendo $m_{r}=\frac{M_{e u}}{f^{\prime}{ }^{*} b^{*} d^{2}}$

tendremos: $m_{r}=0.85^{*} \emptyset^{*} k_{a}^{*}\left(1-k_{a} / 2\right)$

de la ecuación (c):

$$
\begin{aligned}
M_{\text {eu }}= & \varnothing^{*}\left(N_{u} / \varnothing+T_{s}\right)^{\star}(d-a / 2) \quad T_{s}=f_{s}{ }^{*} A_{s} \\
& \left(N_{u}+\varnothing^{*} T_{s}\right)^{*}(d-a / 2)
\end{aligned}
$$

de donde despejamos $A_{s}$ :

$$
A_{s}=\frac{M_{e u}}{\emptyset^{*} f_{s}^{*}(d-a / 2)}-\frac{N_{u}}{\emptyset^{*} f_{s}}
$$

Como $f_{s}=f_{y}$ (la armadura traccionada debe estar en fluencia), $y$ haciendo

$$
\frac{M_{e u}}{\emptyset * \mathrm{f}_{\mathrm{y}} *(\mathrm{~d}-\mathrm{a} / 2)}=\omega * \frac{f^{\prime}{ }_{c}}{f_{y}} * b^{*} d
$$

tendremos: $A_{s}=\omega^{*}\left(f_{c}^{\prime} / f_{y}\right)^{*} b^{*} d-N_{u} /\left(\varnothing^{*} f_{y}\right)$

$\omega$ : cuantía mecánica

$$
\begin{aligned}
\omega & =\frac{M_{e u}{ }^{*} f_{y}}{\emptyset^{*} f_{y} *(d-a / 2)^{*} f^{\prime}{ }^{*}{ }^{*} b^{*} d} \\
\omega & =\frac{M_{e u}}{\emptyset^{*} \mathrm{f}_{\mathrm{c}}{ }^{*} \mathrm{~b}^{*} \mathrm{~d}^{2 *}\left(1-\mathrm{k}_{\mathrm{a}} / 2\right)} \quad a=\mathrm{k}_{\mathrm{a}}{ }^{* d}
\end{aligned}
$$




$$
\begin{aligned}
& \frac{M_{e u}}{f^{\prime}{ }^{*}{ }^{*} b^{*} d^{2}}=m_{r}=0.85 * \emptyset * \mathrm{k}_{\mathrm{a}} *\left(1-\mathrm{k}_{\mathrm{a}} / 2\right) \\
& \omega=0.85^{*} \mathrm{k}_{\mathrm{a}}
\end{aligned}
$$

Podemos encontrar una relación directa entre $\mathrm{m}_{\mathrm{r}}$ y $\omega$ despejando $k_{a}$ de la ecuación $m_{r}=0.85^{*} \varnothing^{*} k_{a}^{*}\left(1-k_{a} / 2\right)$

$$
\begin{aligned}
& \mathrm{m}_{\mathrm{r}}=0.85^{*} \varnothing^{*} \mathrm{k}_{\mathrm{a}}-0.85^{\star} \varnothing^{*} \mathrm{k}_{\mathrm{a}}{ }^{2 / 2} \\
& k_{a}=1-\sqrt{1-\frac{m_{r}}{0.425^{*} \varnothing}} \quad(\text { Ecuación 4.3) }
\end{aligned}
$$

por tanto:

$$
\omega=0.85 *\left[1-\sqrt{1-\frac{m_{r}}{0.425 * \varnothing}}\right]
$$

Se puede notar que los valores de $\omega, \emptyset, \mathrm{m}_{\mathrm{r}} \mathrm{y} \mathrm{k}_{\mathrm{a}}$ dependen de $\varepsilon_{\mathrm{s}}$, por lo que se puede construir una tabla con estos valores y usarla para diseño. (Ver tablas anexas)

Si se va a hacer un programa o se va a hacer el diseño a mano, se debe usar un proceso iterativo:

[1] Suponer que $\varnothing=0.90$

[2] Calcular $\mathrm{k}_{\mathrm{a}}$ a partir de $\varnothing$ y $\mathrm{m}_{\mathrm{r}} \quad$ (Ecuación 4.3)

[3] Con $k_{a}$ y $\beta_{1}$ calcular $e_{s}$ (Ecuación 4.1 y 4.2 )

[4] Con $\varepsilon_{\mathrm{s}}$ calcular el nuevo valor de $\varnothing$

[5] Si el nuevo valor de $\varnothing$ es diferente del valor inicial, volver al paso [2] 
Una forma de saber de antemano cual es el valor de $\emptyset$ es calcular el valor del momento reducido $\mathrm{m}_{\mathrm{r}}$ para el estado de deformación $\varepsilon_{\mathrm{s}}=0.005$. Para definir este estado usaremos el subíndice " 5 ".

$$
\begin{aligned}
\varepsilon_{\mathrm{s}} & =0.005 \\
\mathrm{k}_{\mathrm{c} 5} & =0.003 /(0.003+0.005)=0.375 \\
\mathrm{k}_{\mathrm{a} 5} & =\beta_{1}{ }^{*} k_{\mathrm{c} 5}=0.375^{\star} \beta_{1} \\
\varnothing_{5} & =0.90 \\
m_{\mathrm{r} 5} & =0.85^{*} 0.90^{*} 0.375^{\star} \beta_{1}{ }^{*}\left(1-0.375^{\star} \beta_{1} / 2\right) \\
& =0.2869^{*} \beta_{1}{ }^{*}\left(1-0.1875^{\star} \beta_{1}\right)
\end{aligned}
$$

\begin{tabular}{|r|r|r|}
\hline$f ' c$ & $\beta_{1}$ & $\mathrm{~m}_{\mathrm{r} 5}$ \\
\hline$\left[\mathrm{kg} / \mathrm{cm}^{2}\right]$ & {$[-]$} & {$[-]$} \\
$\leq 280$ & 0.85 & 0.205 \\
350 & 0.80 & 0.195 \\
420 & 0.75 & 0.185 \\
500 & 0.69 & 0.173 \\
560 & 0.65 & 0.164 \\
\hline
\end{tabular}

cuando se tenga $m_{r}=m_{r s}$ siempre tendremos $\varnothing=0.90$

Son muy raros los casos donde haya que iterar. Las deformaciones del acero donde el factor $\emptyset$ no es 0.90 son muy pequeñas, lo que corresponde a secciones muy solicitadas. En estos casos generalmente es más económico aumentar el peralte de la sección.

\section{SECCIONES CON ARMADURA DE COMPRESIÓN}

Según $§ 10.3 .5$, para elementos no pretensados con una fuerza axial de compresión menor de $0.10^{\star} \mathrm{f}_{\mathrm{c}}{ }^{\star} \mathrm{A}_{\mathrm{g}}$, la deformación de la armadura mas traccionada $\varepsilon_{1}$ no debe ser menor de 0.004. Esta deformación del acero corresponde a la zona $\mathrm{B}$, o sea la zona de transición. 
Usando el subíndice 1 para definir este estado de deformación, tendremos:

$$
\begin{aligned}
& \varepsilon_{\mathrm{sl}}=0.004 \\
& \mathrm{k}_{\mathrm{cl}}=0.003 /(0.003+0.004)=0.4286 \\
& \mathrm{k}_{\mathrm{al}}=\beta_{1}{ }^{*} \mathrm{k}_{\mathrm{c}}=0.4286^{*} \beta_{1} \\
& \varnothing_{1}=0.65+0.25^{\star}\left(\mathrm{e}_{\mathrm{sl}}-\mathrm{e}_{\mathrm{sb}}\right) /\left(0.005-\mathrm{e}_{\mathrm{sb}}\right) \\
& \mathrm{m}_{\mathrm{rl}}=0.85^{*} \varnothing_{1}{ }^{*} \mathrm{k}_{\mathrm{al}}{ }^{*}\left(1-\mathrm{k}_{\mathrm{al}} / 2\right)
\end{aligned}
$$

El momento máximo que puede resistir la sección sin armadura de compresión será:

$$
M_{\text {eul }}=m_{r i}{ }^{*} f_{c}{ }_{c}{ }^{*} b^{*} d^{2}
$$

o sea que cuando el valor $m_{r}$ sea menor que $m_{r l}$, la sección no necesita armaduras de compresión.

\section{LÍMITE PARA SECCIONES SIN ARMADURAS DE COMPRESIÓN}

$$
\varepsilon_{\mathrm{sl}}=0.004 \quad \mathrm{k}_{\mathrm{cl}}=0.4286
$$

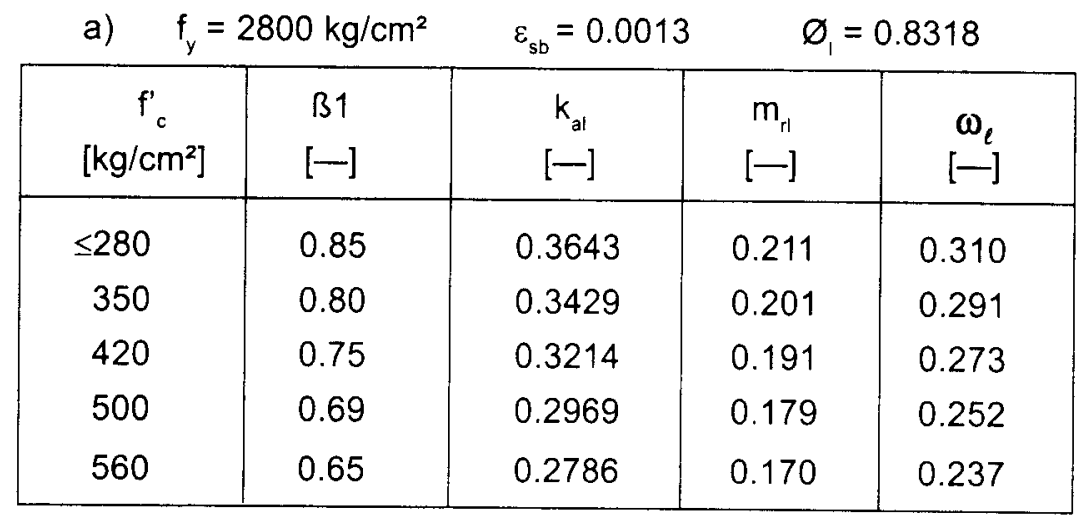


b) $\mathrm{f}_{\mathrm{y}}=4200 \mathrm{~kg} / \mathrm{cm}^{2} \quad \varepsilon_{\mathrm{sb}}=0.0020 \quad \varnothing_{1}=0.8167$

\begin{tabular}{|c|c|c|c|c|}
\hline $\begin{array}{c}f^{\prime}{ }_{c} \\
{\left[\mathrm{~kg} / \mathrm{cm}^{2}\right]}\end{array}$ & $\begin{array}{c}B 1 \\
{[-]}\end{array}$ & $\begin{array}{c}k_{\mathrm{al}} \\
{[-]}\end{array}$ & $\begin{array}{c}\mathrm{m}_{\mathrm{r}} \\
{[-]}\end{array}$ & $\begin{array}{c}\omega_{\ell} \\
{[-]}\end{array}$ \\
\hline$\leq 280$ & 0.85 & 0.3643 & 0.207 & 0.310 \\
350 & 0.80 & 0.3429 & 0.197 & .291 \\
420 & 0.75 & 0.3214 & 0.187 & 0.273 \\
500 & 0.69 & 0.2969 & 0.176 & 0.252 \\
560 & 0.65 & 0.2786 & 0.166 & 0.237 \\
\hline
\end{tabular}

Cuando $M_{e u}>M_{\text {eul }}$ (o lo que es lo mismo: $m_{r}>m_{r l}$ ) el exceso de momento por encima de $\mathrm{M}_{\text {eul }}$ deberá ser resistido por un par de armaduras adicionales en tracción y compresión.

$$
\Delta \mathrm{M}_{\text {eu }}=\mathrm{M}_{\text {eu }}-\mathrm{M}_{\text {eul }}
$$

a) Armadura traccionada:

$$
\Delta A_{s}=\frac{\Delta M_{\text {eu }}}{\varnothing_{\ell}{ }^{*} f_{y}{ }^{*}(d-d)} \quad \text { fs }=f y\left(\varepsilon_{s}=0.004>f_{y} / E_{s}\right)
$$

La armadura traccionada total será:

$$
A_{s}=w_{1}^{*}\left(f_{c}^{\prime} / f_{y}\right)^{*} b^{*} d-N_{u} /\left(\varnothing_{,}^{*} f_{y}\right)+\Delta A_{s}
$$

b) Armadura comprimida:

$$
A_{s}^{\prime}=\frac{\Delta M_{e u}}{\emptyset_{\ell} *\left(\mathrm{f}_{\mathrm{s}}-0.85 * \mathrm{f}_{\mathrm{c}}\right) *\left(\mathrm{~d}-\mathrm{d}^{\prime}\right)}
$$

Se resta $0.85 \mathrm{f}_{\mathrm{c}}{ }_{\mathrm{c}}$ a f' ${ }_{\mathrm{s}}$ para descontar el área ocupada por el acero en la zona comprimida de hormigón. 
El esfuerzo f' de la armadura comprimida depende de $\varepsilon_{s}$, que podemos calcular por relación de triángulos:

$$
\begin{gathered}
\frac{\varepsilon_{s e}-\varepsilon_{c}}{d}=\frac{\varepsilon_{s}^{\prime}-\varepsilon_{c}}{d^{\prime}} \\
\frac{0.004+0.003}{d}=\frac{\varepsilon_{s}^{\prime}+0.003}{d^{\prime}} \\
\varepsilon_{s}^{\prime}=0.007 \frac{d^{\prime}}{d}-0.003
\end{gathered}
$$

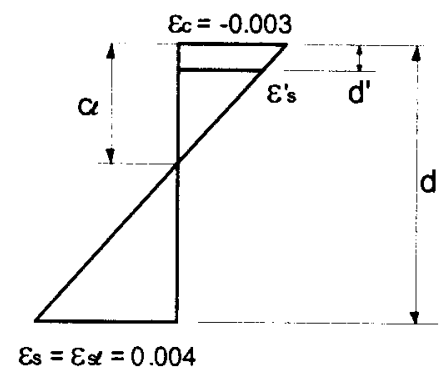

El esfuerzo en la armadura comprimida será:

$$
f^{\prime}=E^{*} \varepsilon_{s}^{\prime} \leq f_{y}
$$

Aun cuando no esta prohibido por el reglamento, en la practica es preferible evitar secciones donde el factor $\varnothing$ sea menor de 0.90 , pues esto da como resultado secciones antieconómicas, al penalizar la resistencia de la sección para compensar la poca ductilidad. Como las secciones con armaduras de compresión siempre caen en este rango, en general es preferible aumentar el peralte de la sección que usar estas secciones. 


\section{ANEXO}

TABLAS PARA EL DISEÑO DE SECCIONES

RECTANGULARES SOMETIDAS A FLEXIÓN

COMPUESTA CON GRAN EXCENTRICIDAD

REGLAMENTO ACI 318-02 


\begin{tabular}{|c|c|c|c|c|c|}
\hline \multicolumn{6}{|c|}{ DISEÑO DE SECCIONES DE HORMIGÓN ARMADO } \\
\hline \multicolumn{3}{|c|}{$f_{y}=2800 \mathrm{~kg} / \mathrm{cm}^{2}$} & \multicolumn{2}{|c|}{$\mathrm{f}_{c}^{\prime}<=280 \mathrm{~kg} / \mathrm{cm}^{2}$} & \\
\hline \multicolumn{3}{|c|}{$\varepsilon_{\mathrm{sb}}=0.0013$} & \multicolumn{2}{|c|}{$ß 1=0.85$} & \\
\hline \multicolumn{3}{|c|}{$\mathrm{k}_{\mathrm{cl}}=0.429$} & \multicolumn{3}{|c|}{$k_{a l}=0.364$} \\
\hline \multicolumn{3}{|c|}{$\varnothing_{1}=0.832$} & \multicolumn{3}{|c|}{$m_{r l}=0.211$} \\
\hline$m_{r}$ & $\varnothing$ & $\mathrm{k}_{\mathrm{a}}$ & $\mathrm{k}_{\mathrm{c}}$ & $\varepsilon_{\mathrm{s}}$ & $\omega$ \\
\hline 0.050 & 0.900 & 0.068 & 0.080 & 0.0347 & 0.058 \\
\hline 0.055 & 0.900 & 0.075 & 0.088 & 0.0311 & 0.063 \\
\hline 0.060 & 0.900 & 0.082 & 0.096 & 0.0282 & 0.070 \\
\hline 0.065 & 0.900 & 0.089 & 0.105 & 0.0257 & 0.076 \\
\hline 0.070 & 0.900 & 0.096 & 0.113 & 0.0235 & 0.082 \\
\hline 0.075 & 0.900 & 0.103 & 0.122 & 0.0217 & 0.088 \\
\hline 0.080 & 0.900 & 0.111 & 0.130 & 0.0200 & 0.094 \\
\hline 0.085 & 0.900 & 0.118 & 0.139 & 0.0186 & 0.100 \\
\hline 0.090 & 0.900 & 0.126 & 0.148 & 0.0173 & 0.107 \\
\hline 0.095 & 0.900 & 0.133 & 0.157 & 0.0162 & 0.113 \\
\hline 0.100 & 0.900 & 0.141 & 0.165 & 0.0151 & 0.120 \\
\hline 0.105 & 0.900 & 0.148 & 0.174 & 0.0142 & 0.126 \\
\hline 0.110 & 0.900 & 0.156 & 0.183 & 0.0134 & 0.133 \\
\hline 0.115 & 0.900 & 0.164 & 0.193 & 0.0126 & 0.139 \\
\hline 0.120 & 0.900 & 0.172 & 0.202 & 0.0119 & 0.146 \\
\hline 0.125 & 0.900 & 0.180 & 0.211 & 0.0112 & 0.153 \\
\hline 0.130 & 0.900 & 0.188 & 0.221 & 0.0106 & 0.159 \\
\hline 0.135 & 0.900 & 0.196 & 0.230 & 0.0100 & 0.166 \\
\hline 0.140 & 0.900 & 0.204 & 0.240 & 0.0095 & 0.173 \\
\hline 0.145 & 0.900 & 0.212 & 0.249 & 0.0090 & 0.180 \\
\hline 0.150 & 0.900 & 0.220 & 0.259 & 0.0086 & 0.187 \\
\hline 0.155 & 0.900 & 0.229 & 0.269 & 0.0081 & 0.494 \\
\hline 0.160 & 0.900 & 0.237 & 0.279 & 0.0077 & 0.202 \\
\hline 0.165 & 0.900 & 0.246 & 0.289 & 0.0074 & 0.209 \\
\hline 0.170 & 0.900 & 0.255 & 0.300 & 0.0070 & 0.216 \\
\hline 0.175 & 0.900 & 0.263 & 0.310 & 0.0067 & 0.224 \\
\hline 0.180 & 0.900 & 0.272 & 0.320 & 0.0064 & 0.232 \\
\hline 0.185 & 0.900 & 0.281 & 0.331 & 0.0061 & 0.239 \\
\hline 0.190 & 0.900 & 0.291 & 0.342 & 0.0058 & 0.247 \\
\hline 0.195 & 0.900 & 0.300 & 0.353 & 0.0055 & 0.255 \\
\hline 0.200 & 0.900 & 0.309 & 0.364 & 0.0052 & 0.263 \\
\hline 0.205 & 0.900 & 0.319 & 0.375 & 0.0050 & 0.271 \\
\hline 0.210 & 0.849 & 0.352 & 0.414 & 0.0043 & 0.299 \\
\hline 0.211 & 0.843 & 0.356 & 0.418 & 0.0042 & 0.302 \\
\hline
\end{tabular}

66 


\begin{tabular}{|c|c|c|c|c|c|}
\hline \multicolumn{6}{|c|}{ DISEÑO DE SECCIONES DE HORMIGÓN ARMADO } \\
\hline \multicolumn{3}{|c|}{$f_{y}=2800 \mathrm{~kg} / \mathrm{cm}^{2}$} & \multicolumn{2}{|c|}{$f_{c}^{\prime}=350 \mathrm{~kg} / \mathrm{cm}^{2}$} & \multirow[b]{5}{*}{$\omega$} \\
\hline \multicolumn{3}{|c|}{$\varepsilon_{\mathrm{sb}}=0.0013$} & \multicolumn{2}{|c|}{$B 1=0.80$} & \\
\hline \multicolumn{3}{|c|}{$\mathrm{k}_{\mathrm{cl}}=0.429$} & \multicolumn{2}{|c|}{$k_{a l}=0.343$} & \\
\hline \multicolumn{2}{|c|}{$\varnothing_{1}=0.832$} & & \multicolumn{2}{|c|}{$\mathrm{m}_{\mathrm{rl}}=0.201$} & \\
\hline$m_{r}$ & $\varnothing$ & $\mathrm{k}_{\mathrm{a}}$ & $\mathrm{k}_{\mathrm{c}}$ & $\varepsilon_{\mathrm{s}}$ & \\
\hline 0.050 & 0.900 & 0.068 & 0.085 & 0.0325 & 0.058 \\
\hline 0.055 & 0.900 & 0.075 & 0.093 & 0.0291 & 0.063 \\
\hline 0.060 & 0.900 & 0.082 & 0.102 & 0.0263 & 0.070 \\
\hline 0.065 & 0.900 & 0.089 & 0.111 & 0.0240 & 0.076 \\
\hline 0.070 & 0.900 & 0.096 & 0.120 & 0.0220 & 0.082 \\
\hline 0.075 & 0.900 & 0.103 & 0.129 & 0.0202 & 0.088 \\
\hline 0.080 & 0.900 & 0.111 & 0.138 & 0.0187 & 0.094 \\
\hline 0.085 & 0.900 & 0.118 & 0.148 & 0.0173 & 0.100 \\
\hline 0.090 & 0.900 & 0.126 & 0.157 & 0.0161 & 0.107 \\
\hline 0.095 & 0.900 & 0.133 & 0.166 & 0.0150 & 0.113 \\
\hline 0.100 & 0.900 & 0.141 & 0.176 & 0.0141 & 0.120 \\
\hline 0.105 & 0.900 & 0.148 & 0.185 & 0.0132 & 0.126 \\
\hline 0.110 & 0.900 & 0.156 & 0.195 & 0.0124 & 0.133 \\
\hline 0.115 & 0.900 & 0.164 & 0.205 & 0.0117 & 0.139 \\
\hline 0.120 & 0.900 & 0.172 & 0.214 & 0.0110 & 0.146 \\
\hline 0.125 & 0.900 & 0.180 & 0.224 & 0.0104 & 0.153 \\
\hline 0.130 & 0.900 & 0.188 & 0.234 & 0.0098 & 0.159 \\
\hline 0.135 & 0.900 & 0.196 & 0.245 & 0.0093 & 0.166 \\
\hline 0.140 & 0.900 & 0.204 & 0.255 & 0.0088 & 0.173 \\
\hline 0.145 & 0.900 & 0.212 & 0.265 & 0.0083 & 0.180 \\
\hline 0.150 & 0.900 & 0.220 & 0.275 & 0.0079 & 0.187 \\
\hline 0.155 & 0.900 & 0.229 & 0.286 & 0.0075 & 0.194 \\
\hline 0.160 & 0.900 & 0.237 & 0.297 & 0.0071 & 0.202 \\
\hline 0.165 & 0.900 & 0.246 & 0.307 & 0.0068 & 0.209 \\
\hline 0.170 & 0.900 & 0.255 & 0.318 & 0.0064 & 0.216 \\
\hline 0.175 & 0.900 & 0.263 & 0.329 & 0.0061 & 0.224 \\
\hline 0.180 & 0.900 & 0.272 & 0.340 & 0.0058 & 0.232 \\
\hline 0.185 & 0.900 & 0.281 & 0.352 & 0.0055 & 0.239 \\
\hline 0.190 & 0.900 & 0.291 & 0.363 & 0.0053 & 0.247 \\
\hline 0.195 & 0.900 & 0.300 & 0.375 & 0.0050 & 0.255 \\
\hline 0.200 & 0.864 & 0.321 & 0.402 & 0.0045 & 0.273 \\
\hline 0.201 & 0.860 & 0.324 & 0.405 & 0.0044 & 0.275 \\
\hline
\end{tabular}




\begin{tabular}{|c|c|c|c|c|c|}
\hline \multicolumn{6}{|c|}{ DISEÑO DE SECCIONES DE HORMIGÓN ARMADO } \\
\hline \multicolumn{2}{|c|}{$f_{y}=2800 \mathrm{~kg} / \mathrm{cm}^{2}$} & \multicolumn{4}{|c|}{$\mathrm{f}_{\mathrm{c}}^{\prime}=420 \mathrm{~kg} / \mathrm{cm}^{2}$} \\
\hline \multicolumn{2}{|c|}{$\varepsilon_{\mathrm{sb}}=0.0013$} & \multicolumn{4}{|c|}{$ß 1=0.75$} \\
\hline \multicolumn{2}{|c|}{$k_{c l}=0.429$} & \multicolumn{4}{|c|}{$k_{a}=0.321$} \\
\hline \multicolumn{2}{|c|}{$\varnothing_{1}=0.832$} & \multicolumn{4}{|c|}{$m_{r l}=0.191$} \\
\hline$m_{r}$ & $\varnothing$ & $\mathrm{k}_{\mathrm{a}}$ & $k_{c}$ & $\varepsilon_{\mathrm{s}}$ & $\omega$ \\
\hline 0.050 & 0.900 & 0.068 & 0.090 & 0.0303 & 0.058 \\
\hline 0.055 & 0.900 & 0.075 & 0.100 & 0.0271 & 0.063 \\
\hline 0.060 & 0.900 & 0.082 & 0.109 & 0.0245 & 0.070 \\
\hline 0.065 & 0.900 & 0.089 & 0.119 & 0.0223 & 0.076 \\
\hline 0.070 & 0.900 & 0.096 & 0.128 & 0.0204 & 0.082 \\
\hline 0.075 & 0.900 & 0.103 & 0.138 & 0.0188 & 0.088 \\
\hline 0.080 & 0.900 & 0.111 & 0.148 & 0.0173 & 0.094 \\
\hline 0.085 & 0.900 & 0.118 & 0.157 & 0.0161 & 0.100 \\
\hline 0.090 & 0.900 & 0.126 & 0.167 & 0.0149 & 0.107 \\
\hline 0.095 & 0.900 & 0.133 & 0.177 & 0.0139 & 0.113 \\
\hline 0.100 & 0.900 & 0.141 & 0.187 & 0.0130 & 0.120 \\
\hline 0.105 & 0.900 & 0.148 & 0.198 & 0.0122 & 0.126 \\
\hline 0.110 & 0.900 & 0.156 & 0.208 & 0.0114 & 0.133 \\
\hline 0.115 & 0.900 & 0.164 & 0.218 & 0.0107 & 0.139 \\
\hline 0.120 & 0.900 & 0.172 & 0.229 & 0.0101 & 0.146 \\
\hline 0.125 & 0.900 & 0.180 & 0.239 & 0.0095 & 0.153 \\
\hline 0.130 & 0.900 & 0.188 & 0.250 & 0.0090 & 0.159 \\
\hline 0.135 & 0.900 & 0.196 & 0.261 & 0.0085 & 0.166 \\
\hline 0.140 & 0.900 & 0.204 & 0.272 & 0.0080 & 0.173 \\
\hline 0.145 & 0.900 & 0.212 & 0.283 & 0.0076 & 0.180 \\
\hline 0.150 & 0.900 & 0.220 & 0.294 & 0.0072 & 0.187 \\
\hline 0.155 & 0.900 & 0.229 & 0.305 & 0.0068 & 0.194 \\
\hline 0.160 & 0.900 & 0.237 & 0.316 & 0.0065 & 0.202 \\
\hline 0.165 & 0.900 & 0.246 & 0.328 & 0.0061 & 0.209 \\
\hline 0.170 & 0.900 & 0.255 & 0.340 & 0.0058 & 0.216 \\
\hline 0.175 & 0.900 & 0.263 & 0.351 & 0.0055 & 0.224 \\
\hline 0.180 & 0.900 & 0.272 & 0.363 & 0.0053 & 0.232 \\
\hline 0.185 & 0.899 & 0.282 & 0.376 & 0.0050 & 0.239 \\
\hline 0.190 & 0.889 & 0.287 & 0.383 & 0.0048 & 0.244 \\
\hline 0.191 & 0.892 & 0.286 & 0.381 & 0.0049 & 0.243 \\
\hline
\end{tabular}




\begin{tabular}{|c|c|c|c|c|c|}
\hline \multicolumn{6}{|c|}{ DISEÑO DE SECCIONES DE HORMIGÓN ARMADO } \\
\hline \multicolumn{2}{|c|}{$f_{y}=4200 \mathrm{~kg} / \mathrm{cm}^{2}$} & \multicolumn{4}{|c|}{$\mathrm{f}_{\mathrm{c}}^{\prime}<=280 \mathrm{~kg} / \mathrm{cm}^{2}$} \\
\hline \multirow{3}{*}{\multicolumn{2}{|c|}{$\begin{array}{l}\varepsilon_{\text {sb }}=0.0020 \\
k_{c l}=0.429 \\
\varnothing I=0.817\end{array}$}} & & \multirow{3}{*}{\multicolumn{3}{|c|}{$\begin{array}{l}B 1=0.85 \\
k_{a l}=0.364 \\
m_{r l}=0.207\end{array}$}} \\
\hline & & & & & \\
\hline & & & & & \\
\hline $\mathrm{m}_{\mathrm{r}}$ & $\varnothing$ & $\mathrm{k}_{\mathrm{a}}$ & $k_{c}$ & $\varepsilon_{\mathrm{s}}$ & $\omega$ \\
\hline 0.050 & 0.900 & 0.068 & 0.080 & 0.0347 & 0.058 \\
\hline 0.055 & 0.900 & 0.075 & 0.088 & 0.0311 & 0.063 \\
\hline 0.060 & 0.900 & 0.082 & 0.096 & 0.0282 & 0.070 \\
\hline 0.065 & 0.900 & 0.089 & 0.105 & 0.0257 & 0.076 \\
\hline 0.070 & 0.900 & 0.096 & 0.113 & 0.0235 & 0.082 \\
\hline 0.075 & 0.900 & 0.103 & 0.122 & 0.0217 & 0.088 \\
\hline 0.080 & 0.900 & 0.111 & 0.130 & 0.0200 & 0.094 \\
\hline 0.085 & 0.900 & 0.118 & 0.139 & 0.0186 & 0.100 \\
\hline 0.090 & 0.900 & 0.126 & 0.148 & 0.0173 & 0.107 \\
\hline 0.095 & 0.900 & 0.133 & 0.157 & 0.0162 & 0.113 \\
\hline 0.100 & 0.900 & 0.141 & 0.165 & 0.0151 & 0.120 \\
\hline 0.105 & 0.900 & 0.148 & 0.174 & 0.0142 & 0.126 \\
\hline 0.110 & 0.900 & 0.156 & 0.183 & 0.0134 & 0.133 \\
\hline 0.115 & 0.900 & 0.164 & 0.193 & 0.0126 & 0.139 \\
\hline 0.120 & 0.900 & 0.172 & 0.202 & 0.0119 & 0.146 \\
\hline 0.125 & 0.900 & 0.180 & 0.211 & 0.0112 & 0.153 \\
\hline 0.130 & 0.900 & 0.188 & 0.221 & 0.0106 & 0.159 \\
\hline 0.135 & 0.900 & 0.196 & 0.230 & 0.0100 & 0.166 \\
\hline 0.140 & 0.900 & 0.204 & 0.240 & 0.0095 & 0.173 \\
\hline 0.145 & 0.900 & 0.212 & 0.249 & 0.0090 & 0.180 \\
\hline 0.150 & 0.900 & 0.220 & 0.259 & 0.0086 & 0.187 \\
\hline 0.155 & 0.900 & 0.229 & 0.269 & 0.0081 & 0.194 \\
\hline 0.160 & 0.900 & 0.237 & 0.279 & 0.0077 & 0.202 \\
\hline 0.165 & 0.900 & 0.246 & 0.289 & 0.0074 & 0.209 \\
\hline 0.170 & 0.900 & 0.255 & 0.300 & 0.0070 & 0.216 \\
\hline 0.175 & 0.900 & 0.263 & 0.310 & 0.0067 & 0.224 \\
\hline 0.180 & 0.900 & 0.272 & 0.320 & 0.0064 & 0.232 \\
\hline 0.185 & 0.900 & 0.281 & 0.331 & 0.0061 & 0.239 \\
\hline 0.190 & 0.900 & 0.291 & 0.342 & 0.0058 & 0.247 \\
\hline 0.195 & 0.900 & 0.300 & 0.353 & 0.0055 & 0.255 \\
\hline 0.200 & 0.900 & 0.309 & 0.364 & 0.0052 & 0.263 \\
\hline 0.205 & 0.899 & 0.319 & 0.375 & 0.0050 & $0: 271$ \\
\hline 0.207 & 0.817 & 0.364 & 0.429 & 0.0040 & 0.310 \\
\hline
\end{tabular}




\begin{tabular}{|c|c|c|c|c|c|}
\hline \multicolumn{6}{|c|}{ DISEÑO DE SECCIONES DE HORMIGÓN ARMADO } \\
\hline \multicolumn{3}{|c|}{$f_{y}=4200 \mathrm{~kg} / \mathrm{cm}^{2}$} & \multicolumn{3}{|c|}{$\mathrm{f}_{c}^{\prime}=350 \mathrm{~kg} / \mathrm{cm}^{2}$} \\
\hline \multicolumn{3}{|c|}{$\varepsilon_{\mathrm{sb}}=0.0020$} & \multicolumn{2}{|c|}{$B 1=0.80$} & \multirow[b]{4}{*}{$\omega$} \\
\hline & 0.429 & & \multicolumn{2}{|c|}{$\mathrm{k}_{\mathrm{a}}=0.343$} & \\
\hline \multicolumn{2}{|c|}{$\varnothing_{1}=0.817$} & & \multicolumn{2}{|c|}{$m_{r l}=0.197$} & \\
\hline$m_{r}$ & $\varnothing$ & $k_{a}$ & $\mathrm{k}_{\mathrm{c}}$ & $\varepsilon_{\mathrm{s}}$ & \\
\hline 0.050 & 0.900 & 0.068 & 0.085 & 0.0325 & 0.058 \\
\hline 0.055 & 0.900 & 0.075 & 0.093 & 0.0291 & 0.063 \\
\hline 0.060 & 0.900 & 0.082 & 0.102 & 0.0263 & 0.070 \\
\hline 0.065 & 0.900 & 0.089 & 0.111 & 0.0240 & 0.076 \\
\hline 0.070 & 0.900 & 0.096 & 0.120 & 0.0220 & 0.082 \\
\hline 0.075 & 0.900 & 0.103 & 0.129 & 0.0202 & 0.088 \\
\hline 0.080 & 0.900 & 0.111 & 0.138 & 0.0187 & 0.094 \\
\hline 0.085 & 0.900 & 0.118 & 0.148 & 0.0173 & 0.100 \\
\hline 0.090 & 0.900 & 0.126 & 0.157 & 0.0161 & 0.107 \\
\hline 0.095 & 0.900 & 0.133 & 0.166 & 0.0150 & 0.113 \\
\hline 0.100 & 0.900 & 0.141 & 0.176 & 0.0141 & 0.120 \\
\hline 0.105 & 0.900 & 0.148 & 0.185 & 0.0132 & 0.126 \\
\hline 0.110 & 0.900 & 0.156 & 0.195 & 0.0124 & 0.133 \\
\hline 0.115 & 0.900 & 0.164 & 0.205 & 0.0117 & 0.139 \\
\hline 0.120 & 0.900 & 0.172 & 0.214 & 0.0110 & 0.146 \\
\hline 0.125 & 0.900 & 0.180 & 0.224 & 0.0104 & 0.153 \\
\hline 0.130 & 0.900 & 0.188 & 0.234 & 0.0098 & 0.159 \\
\hline 0.135 & 0.900 & 0.196 & 0.245 & 0.0093 & 0.166 \\
\hline 0.140 & 0.900 & 0.204 & 0.255 & 0.0088 & 0.173 \\
\hline 0.145 & 0.900 & 0.212 & 0.265 & 0.0083 & 0.180 \\
\hline 0.150 & 0.900 & 0.220 & 0.275 & 0.0079 & 0.187 \\
\hline 0.155 & 0.900 & 0.229 & 0.286 & 0.0075 & 0.194 \\
\hline 0.160 & 0.900 & 0.237 & 0.297 & 0.0071 & 0.202 \\
\hline 0.165 & 0.900 & 0.246 & 0.307 & 0.0068 & 0.209 \\
\hline 0.170 & 0.900 & 0.255 & 0.318 & 0.0064 & 0.216 \\
\hline 0.175 & 0.900 & 0.263 & 0.329 & 0.0061 & 0.224 \\
\hline 0.180 & 0.900 & 0.272 & 0.340 & 0.0058 & 0.232 \\
\hline 0.185 & 0.900 & 0.281 & 0.352 & 0.0055 & 0.239 \\
\hline 0.190 & 0.900 & 0.291 & 0.363 & 0.0053 & 0.247 \\
\hline 0.195 & 0.900 & 0.300 & 0.375 & 0.0050 & 0.255 \\
\hline 0.197 & 0.817 & 0.343 & 0.429 & 0.0040 & 0.291 \\
\hline
\end{tabular}




\begin{tabular}{|c|c|c|c|c|c|}
\hline \multicolumn{6}{|c|}{ DISEÑO DE SECCIONES DE HORMIGÓN ARMADO } \\
\hline \multicolumn{2}{|c|}{$f_{y}=4200 \mathrm{~kg} / \mathrm{cm}^{2}$} & \multicolumn{4}{|c|}{$\mathrm{f}_{\mathrm{c}}^{\prime}=420 \mathrm{~kg} / \mathrm{cm}^{2}$} \\
\hline \multirow{3}{*}{\multicolumn{2}{|c|}{$\begin{array}{l}\varepsilon_{\mathrm{sb}}=0.0020 \\
\mathrm{k}_{\mathrm{cl}}=0.429 \\
\varnothing_{1}=0.817\end{array}$}} & \multicolumn{4}{|c|}{$ß 1=0.75$} \\
\hline & & \multicolumn{4}{|c|}{$\mathrm{k}_{\mathrm{al}}=0.321$} \\
\hline & & \multicolumn{4}{|c|}{$m_{r 1}=0.187$} \\
\hline $\mathrm{m}_{\mathrm{r}}$ & $\varnothing$ & $\mathrm{k}_{\mathrm{a}}$ & $k_{c}$ & $\varepsilon_{\mathrm{s}}$ & $\omega$ \\
\hline 0.050 & 0.900 & 0.068 & 0.090 & 0.0303 & 0.058 \\
\hline 0.055 & 0.900 & 0.075 & 0.100 & 0.0271 & 0.063 \\
\hline 0.060 & 0.900 & 0.082 & 0.109 & 0.0245 & 0.070 \\
\hline 0.065 & 0.900 & 0.089 & 0.119 & 0.0223 & 0.076 \\
\hline 0.070 & 0.900 & 0.096 & 0.128 & 0.0204 & 0.082 \\
\hline 0.075 & 0.900 & 0.103 & 0.138 & 0.0188 & 0.088 \\
\hline 0.080 & 0.900 & 0.111 & 0.148 & 0.0173 & 0.094 \\
\hline 0.085 & 0.900 & 0.118 & 0.157 & 0.0161 & 0.100 \\
\hline 0.090 & 0.900 & 0.126 & 0.167 & 0.0149 & 0.107 \\
\hline 0.095 & 0.900 & 0.133 & 0.177 & 0.0139 & 0.113 \\
\hline 0.100 & 0.900 & 0.141 & 0.187 & 0.0130 & 0.120 \\
\hline 0.105 & 0.900 & 0.148 & 0.198 & 0.0122 & 0.126 \\
\hline 0.110 & 0.900 & 0.156 & 0.208 & 0.0114 & 0.133 \\
\hline 0.115 & 0.900 & 0.164 & 0.218 & 0.0107 & 0.139 \\
\hline 0.120 & 0.900 & 0.172 & 0.229 & 0.0101 & 0.146 \\
\hline 0.125 & 0.900 & 0.180 & 0.239 & 0.0095 & 0.153 \\
\hline 0.130 & 0.900 & 0.188 & 0.250 & 0.0090 & 0.159 \\
\hline 0.135 & 0.900 & 0.196 & 0.261 & 0.0085 & 0.166 \\
\hline 0.140 & 0.900 & 0.204 & 0.272 & 0.0080 & 0.173 \\
\hline 0.145 & 0.900 & 0.212 & 0.283 & 0.0076 & 0.180 \\
\hline 0.150 & 0.900 & 0.220 & 0.294 & 0.0072 & 0.187 \\
\hline 0.155 & 0.900 & 0.229 & 0.305 & 0.0068 & 0.194 \\
\hline 0.160 & 0.900 & 0.237 & 0.316 & 0.0065 & 0.202 \\
\hline 0.165 & 0.900 & 0.246 & 0.328 & 0.0061 & 0.209 \\
\hline 0.170 & 0.900 & 0.255 & 0.340 & 0.0058 & 0.216 \\
\hline 0.175 & 0.900 & 0.263 & 0.351 & 0.0055 & 0.224 \\
\hline 0.180 & 0.900 & 0.272 & 0.363 & 0.0053 & 0.232 \\
\hline 0.185 & 0.896 & 0.283 & 0.377 & 0.0050 & 0.240 \\
\hline 0.187 & 0.817 & 0.321 & 0.429 & 0.0040 & 0.273 \\
\hline
\end{tabular}

